\title{
Klimawandel, Klimaethik und das drohende Ende der Menschheit
}

Trotz aller wissenschaftlichen Erforschung des Klimas, deren Umfang in den zurückliegenden Jahren immens zugenommen hat, kann zur Stunde niemand mit Sicherheit sagen, ob sich das Klima unseres Planeten in der näheren Zukunft deutlich verändern wird. Auch ist weiterhin umstritten, ob die Ursachen der potentiellen Veränderungen mit der Art des menschlichen Wirtschaftens und des Lebensstils industrialisierter Gesellschaften im 19., 20. und 21. Jahrhundert zu tun haben. Doch wer angesichts der wissenschaftlichen Befunde, Modellrechnungen und beobachtbaren Indizien, die inzwischen vorliegen, heute behauptet, es gäbe keinen hinreichenden Grund, Wirtschaft, Gesellschaft und Politik auf die Möglichkeit einer deutlichen Veränderung des Klimas einzustellen, gleicht einem Autofahrer, der mit hohem Tempo durch den dichten Nebel rast und keinen Grund für die Vermutung sieht, dass vor ihm eine Kurve liegt.

Zwar ist es richtig, was die sogenannten Klimaskeptiker zuweilen sagen: Falls sich das Klima in den nächsten Jahrzehnten gar nicht deutlich verändern sollte oder die Ursachen der potentiellen Veränderungen auf natürliche Faktoren zurückgehen, auf die wir durch wirtschaftliches und politisches Handeln kaum nennenswerten Einfluss nehmen können, scheint jede Investition in den Klimaschutz oder in Maßnahmen der Anpassung an die kommenden Veränderungen auf den ersten Blick eine Verschwendung von Geld, Kraft und Aufmerksamkeit zu sein. Diese Ressourcen sollten in dem Fall besser zur Lösung anderer Probleme verwendet werden. Aber erstens gibt es gute Gründe für die These, dass angemessene und klug durchdachte Investitionen in den Klimaschutz zugleich auch der Lösung manch anderer Probleme von globalem Ausmaß dienen. So könnte der Kampf gegen die bedrückende Armut vieler Menschen auf diesem Planeten mit dem Kampf gegen den Wandel des Klimas verknüpft werden. Klimapolitik ginge auch einher mit der Umstellung der bisherigen Produktion auf eine nachhaltige Energieversorgung, die ohnehin ein Gebot der Stunde ist. Und zweitens ist das Problem, das unter dem Titel Klimawandel firmiert, derart brenzlich, dass sich die Frage schon stellt, ob man nicht besser das Risiko gewaltiger Fehlinvestitionen in Kauf nehmen sollte, statt die Hände angesichts der unklaren Datenlage in den Schoß zu legen. Um im Bild zu bleiben: Falls 
vor uns eine Kurve liegt, sind wir momentan viel zu schnell unterwegs, um Aussichten darauf zu haben, den drohenden Unfall zu überleben.

Um diesen letzten Punkt zu verdeutlichen, ist es ratsam, sich vor Augen zu führen, dass die leider schon fest etablierte Rede vom Klimawandel in zweierlei Hinsichten ein unglücklich gewählter Euphemismus ist. Zum einen verschleiert das neutrale Wort „Wandel“, dass es mit einer nicht zu unterschätzenden Wahrscheinlichkeit eben nicht einfach nur um eine Veränderung des Klimas auf die eine oder andere Weise geht. Es geht um eine katastrophale Veränderung, die nicht nur Milliarden von Menschen ein erbärmliches Leben bereiten kann, sondern mit einer - wieder nicht zu unterschätzenden Wahrscheinlichkeit zum Ende der Menschheit und zahlloser anderer höherer Lebensformen führen wird. Es geht mit anderen Worten um eine drohende Klimakatastrophe im globalen Ausmaß.

Zum anderen lenkt das Wort „Klima“ die Gedanken allzu leicht auf die verkürzte Vorstellung, es gehe primär um eine Veränderung der globalen und regionalen Wetterlagen, um eine $\mathrm{Zu}$ - oder Abnahme von Niederschlagsmengen, um eine Verkürzung oder Verlängerung dieser oder jener Jahreszeit. Aber natürlich reden wir hier nicht vom Wetter. Wir reden über die Gesamtheit der natürlichen Bedingungen der Möglichkeit des Überlebens der Menschheit. Es ist bekannt, was uns nach einer weithin geteilten Einschätzung vieler Klimaforscher im schlimmsten Fall droht, wenn die durchschnittliche Temperatur der Erde in den nächsten Jahrzehnten um mehr als zwei Grad Celsius ansteigt: „Mitglieder des Hadley Centres und andere behaupten, dass wir bei einer Erwärmung über zwei Grad hinaus Kipppunkte überschreiten würden, und dies würde eine Eigendynamik nach sich ziehen, die automatisch zu einer Erwärmung um sechs und mehr Grad führe. Die ersten beiden Kipppunkte seien durch den Kollaps des Amazonas-Regenwaldes und anderer Regenwälder und durch die Freisetzung von $\mathrm{CO}_{2}$ aus dem Boden durch die Wärme aktiver Bakterien beschreibbar. [...] Die Überschreitung auch nur eines dieser Kipppunkte bringe uns automatisch so viel zusätzliches $\mathrm{CO}_{2}$, dass der nächste, das weitgehende Auftauen der Parmafrostböden, erreicht werde. [...] Überschreiten wir zwei Grad, landen wir unaufhaltbar bei sechs oder mehr Grad, so die These von Peter Cox und anderen [...]. Sobald erst einmal der erste überschritten sei, werde das System von Kipppunkten fallen, wie Dominosteine" (Gesang 2011: 40).

Wir können nur hoffen, dass diese Dominothese falsch ist. Denn dass die globale Erwärmung in den kommenden Jahrzehnten unter zwei Grad zu halten sein wird, behauptet heute niemand mehr, der weiß, wovon er spricht. Und eine Erwärmung der Erde um sechs oder noch mehr Grad bedeutet nicht einfach nur heißere Sommer zu Hause auf dem Balkon: „Die wichtigsten Risiken betreffen den Anstieg des Meeresspiegels insbesondere dann, wenn große Abschmelzungsprozesse auf Grön- 
land und in der Antarktis einsetzen würden, eine Abschwächung der thermohalinen Zirkulation („Golfstrom“) und der Monsunniederschläge, eine Zunahme extremer Wetterereignisse mit hohen Schadenspotenzialen, wachsende Wasserknappheit in semiariden und ariden Gebieten, negativer Einfluss auf die Ernährungssicherheit der armen Bevölkerungsgruppen, Verbreitung von Krankheiten (Malaria), steigende Zahl der Klimaflüchtlinge, Sicherheitsrisiken und nicht zuletzt ein beschleunigtes Schwinden der biologischen Vielfalt des Planeten“ (Ott 2009: 60).

Die Politik hat in den zurückliegenden Jahren damit begonnen, die Möglichkeit dieses Horrorszenarios ernst zu nehmen. Bekanntlich zwar nicht ernst genug, sofern es momentan nicht danach aussieht, als ob es nach dem Auslaufen des Kyoto-Protokolls in diesem Jahr in absehbarer Zukunft zu einer neuen internationalen Vereinbarung über eine Einschränkung der globalen Emission von Kohlendioxid und anderen Treibhausgasen kommt. Aber immerhin: Der Klimawandel steht auf einem der oberen Posten der Agenda vieler politischer Akteure, auch wenn deren Aufmerksamkeit und Arbeitszeit derzeit fast ausschließlich durch die europäische und weltweite Finanzkrise gebunden zu sein scheint. Fast als gälte es, diese Ablenkung der Politik von der dringlichen Aufgabe, schnell, effektiv und konzertiert auf den Klimawandel zu reagieren, irgendwie zu kompensieren, hebt seit wenigen Jahren ein neuer Boom in der Angewandten Ethik an: Die zeitgenössische Moralphilosophie ist offenbar mit der Bioethik fertig, die vor einigen Jahren aus dem Schoß der Medizinethik geboren wurde und schnell zu einer Lawine von Publikationen führte. Jetzt gebiert die Umweltethik eine neue Subdisziplin der Angewandten Ethik namens Klimaethik. ${ }^{1}$

Noch ist nicht recht klar, was die Klimaethik eigentlich ist bzw. was sie zu einer eigenständigen Teildisziplin der Angewandten Ethik machen könnte. Auch ist unklar, was man sich von dieser neuen Wissenschaftsdisziplin versprechen kann. Die Mehrzahl der Pioniere auf diesem akademischen Feld sind Vertreter bestimmter Theorien der Gerechtigkeit. Diese Theorien der Gerechtigkeit standen im Zentrum der allgemeinen, oft normativ genannten Moralphilosophie der vier Jahrzehnte, die vergangen sind, seit der Harvard-Philosoph John Rawls mit seiner 1971 erschienenen Monographie $A$ Theory of Justice zuerst den angelsächsischen, dann auch den deutschsprachigen Moraldiskurs neu ausgerichtet hat. Rawls gilt gemeinhin als Egalitarist, also als Vertreter des Standpunkts, dass im Zentrum des Begriffs der Gerechtigkeit das vielschichtige Konzept der Gleichheit steht. Aus egalitaristischer

1 Einen Überblick liefern O'Brien/Clair/Kristoffersen 2010 und Gardiner u. a. 2010. Gesang 2011 ist die bisher einzige Monographie in deutscher Sprache zum Thema. Vergleiche auch Wallacher/ Scharpenseel 2009. 
Sicht muss daher jede adäquate Theorie der Gerechtigkeit klären, welche Rolle die Gleichheit in einer gerechten Gesellschaft spielt und entlang welcher Parameter die Mitglieder solcher Gesellschaften einander gleich sein sollten.

Die Philosophie wäre nicht die Philosophie, wie wir sie kennen, wenn es nicht auch Kritiker des Egalitarismus gäbe. ${ }^{2}$ Diese Non-Egalitaristen bezweifeln, dass Gerechtigkeit in einer gehaltvollen Art und Weise auf irgendeiner Form von Gleichheit beruht. Prominent ist im Lager dieser Theoretiker die Ansicht, dass man das Ausmaß der Gerechtigkeit in einer Gesellschaft nicht erkenne, indem man den ökonomischen, sozialen oder politischen Status ihrer Mitglieder vergleicht, um zu klären, wie gleich oder ungleich diese Menschen einander in dieser oder jener Hinsicht sind. Gerechtigkeit ist in den Augen dieser Philosophen mit anderen Worten kein relationales Konzept. Man erkenne das Ausmaß der Gerechtigkeit in einer Gesellschaft vielmehr dadurch, dass man sich jedes Individuum isoliert vor Augen führt, um die Frage zu klären, ob dieser Mensch von den wichtigen Dingen des Lebens genug hat, um ein anständiges, menschenwürdiges bzw. gutes Leben zu führen. Selbst wer deutlich weniger hat als andere Menschen, so der Kern dieses Standpunkts, mag trotzdem genug haben, um ein gutes Leben führen zu können, wenn sein Status über einem bestimmten Schwellenwert liegt. Dass die Menschen genug haben, sei folglich das zentrale Gebot der Gerechtigkeit. Ob sie gleichviel oder weniger haben als andere Leute, sei hingegen gerechtigkeitstheoretisch irrelevant.

Rawls entwickelte seine Theorie der Gerechtigkeit mit Blick auf eine idealisierte, nationalstaatlich organisierte Gesellschaft, die durch so manches Detail verrät, dass ihm nordamerikanische und einige mitteleuropäische Gesellschaften unserer Gegenwart als Blaupause dienten. Eine Reihe seiner Schüler gingen hingegen dazu über, die zentralen Thesen der Theorie von Rawls auf die globalen Realitäten zu reflektieren, um Theorien der gerechten Ressourcenverteilung im Weltmaßstab und einer gerechten Weltwirtschaftsordnung zu entwickeln. ${ }^{3}$ Wissenschaftliche Publikationen zum Stichwort Globale Gerechtigkeit füllen heute bestimmt mindestens so viele Regale universitärer Bibliotheken, wie es die Arbeiten von Rawls, seinen Verteidigern und Weiterentwicklern zusammen mit den Schriften seiner Kritiker tun.

Was sich derzeit als erster Mainstream der neugeborenen Klimaethik abzeichnet, liest sich zum beträchtlichen Teil als Verlängerung der etablierten Diskussion über die globale Gerechtigkeit. Ging es in der etwas älteren Debatte nicht zuletzt um die Frage, was und wie viel die reicheren Staaten (bzw. deren Bürgerinnen und Bürger)

2 Für eine Übersicht siehe Krebs 2000.

3 Für einen Überblick siehe Pogge/Moellendorf 2008. 
den Bewohnern ärmerer Länder gerechterweise abzugeben hätten, geht es in der klimaethischen Debatte zusätzlich um die Frage, in welchem Zustand die derzeit lebenden und ihnen unmittelbar nachfolgenden Generationen den Planeten Erde zukünftigen Generationen gerechterweise zu hinterlassen haben. ${ }^{4}$ Warum sich diese Frage stellt, liegt auf der Hand: Wenn der Klimawandel Realität ist und wir nichts oder nicht alles uns Mögliche tun, um ihn auf ein erträgliches Maß zu beschränken, berauben wir einige der zukünftigen Generationen der Mittel, die sie bräuchten, um ein anständiges, menschenwürdiges bzw. gutes Leben zu führen. Unzählige weitere Generationen werden unter Umständen wegen uns erst gar nicht das Licht der Welt erblicken, da wir drauf und dran sind, den Planeten unbewohnbar zu machen. Wir Heutigen leben, kurz und drastisch gesagt, auf Kosten von Menschen, die nicht einmal die Chance haben, sich gegen uns zu wehren, da es sie noch gar nicht gibt.

Die Philosophie wäre nicht die Philosophie, wie wir sie kennen, wenn nicht mit raffiniert ersonnenen Gedankenexperimenten und Argumenten um die Frage gestritten würde, ob man von noch nicht existenten Individuen behaupten kann, dass sie moralische Rechte haben, denen Pflichten korrespondieren, die wir hier und heute lebenden Menschen ihnen gegenüber haben. Sind wir in dieser Stunde moralisch dazu verpflichtet, zukünftigen Generationen einen Planeten zu hinterlassen, der nicht zu heiß ist, um ein menschenwürdiges Leben zu führen? Sind wir verpflichtet, unter Umständen auf Wohlstand und weiteren Fortschritt zu verzichten, weil wir sonst unseren Enkeln und Enkelsenkeln nicht den Anteil der Weltressourcen abgeben, der ihnen gerechterweise zusteht?

Im Zusammenhang mit der Klimawandelthematik stellen sich aber auch weitere gerechtigkeitstheoretische Fragen. Wenn die weithin geteilten Annahmen zutreffen, dass ein nicht zu unterschätzender Anteil der globalen Erderwärmung eine kausale Folge der Wirtschafts- und Lebensform industrialisiert-technisierter Gesellschaften ist und dass die katastrophalen Folgen des Klimawandels zuerst und vor allem Menschen in Regionen der Erde treffen werden, die keine Mitglieder dieser Gesellschaften sind, dann stellt sich die Frage, was wir gerechterweise als Nutznießer der technischen Zivilisation den Opfern unseres Nutzens schulden. Müssen wir zum Beispiel unsere Wirtschaftsleistung drastisch drosseln, um Schadstoffemissionen zu vermeiden, damit Entwicklungs- und Schwellenländer ihre Wirtschaftssysteme ausbauen können, ohne dass das globale Klima kollabiert?

4 Fragen der sogenannten Generationen- bzw. intergenerativen Gerechtigkeit wurden ansatzweise auch schon in der genannten Monographie von John Rawls diskutiert. Über den Stand der Debatte informiert Meyer 2008. 
All diese Fragen, die leicht um weitere Problemstellungen ergänzt werden könnten, geben in ihrer Summe zu erkennen, dass es dem eingangs angedeutetem Zweifel zum Trotz sehr wohl ein hinlänglich klar umgrenztes, moralphilosophisches Forschungsfeld gibt, das die eigenständige Bezeichnung Klimaethik verdient. Der Tag ist daher vermutlich nicht fern, an dem in einem der einschlägigen Wissenschaftsverlage die erste Einführung in die Klimaethik erscheinen wird. ${ }^{5}$ Es ist aber auch leicht zu antizipieren, was den Leser dieser Einführung erwarten wird. Nach einer kurzen Einleitung, die ihn über die Neuigkeit, Aktualität und Wichtigkeit der jungen Klimaethik informiert, wird er einen Durchgang durch die wichtigsten Spielarten der egalitaristischen und non-egalitaristischen Gerechtigkeitstheorien vor Augen geführt bekommen, dem dann eine Diskussion der Frage folgt, welche unterschiedlichen Klimaschutzforderungen sich aus den unterschiedlichen Gerechtigkeitskonzeptionen ableiten lassen. Darlegungen dieser Art sind natürlich sinnvoll und hilfreich. Angewandte Ethik leistet ja selten mehr als eben Aufschluss darüber zu vermitteln, welche Konsequenzen aus welchen Annahmen fließen. Aber kein Vertreter der Angewandten Ethik im Allgemeinen und der Klimaethik im Besonderen wird seinen Lesern beweisen können, dass die von ihm favorisierten Annahmen und Konsequenzen die einzigen sind, die sich mit guten Argumenten stützen lassen. Der faktisch beobachtbare Pluralismus auf dem Feld der allgemeinen Gerechtigkeitstheorien wird folglich einen korrespondierenden Pluralismus in der Klimaethik nach sich ziehen.

Diese Beobachtung, dass eine klimaethische Fachdiskussion keinen wissenschaftlichen Konsens unter den Beteiligten in Aussicht stellt, führt mich zu der zweiten der beiden oben angerissenen Fragen: Was kann man sich sinnvollerweise von der Klimaethik versprechen? Was bringt die Klimaethik als eigenständige Teildisziplin der Angewandten Ethik?

Es wäre sicherlich naiv zu glauben, dass wir den Klimawandel allein dadurch vermeiden, dass wir Klimaethik betreiben. Klimaschutz ist Aufgabe politischen Handelns im großen Maßstab und findet nicht im akademischen Rahmen statt. Auch kann es nicht das primäre Ziel sein, durch eine klimaethische Diskussion möglichst viele Menschen zu einer umweltbewussteren Lebensform zu bekehren. Mehr Umweltbewusstsein auf der individuellen Ebene wäre zwar mehr als begrüßenswert. Aber Klimaschutz in dem Ausmaß, das erforderlich ist, um die drohende Katastrophe abzuwenden, ist durch weniger Autofahren, weniger Flugreisen, sparsames Heizen, Fleischverzicht usw. allein nicht zu bewerkstelligen. Die globale Strom-

5 Zwar nicht dem Titel, aber doch dem Inhalt nach liegt zumindest der erste Handbucheintrag bereits vor; siehe Edenhofer/Kowarsch 2011. 
versorgung muss radikal auf erneuerbare Energien umgestellt werden; die durch die Industrie verursachten Schadstoffemissionen müssen drastisch gesenkt werden; die stetige Zunahme der Weltbevölkerung muss aufhören usw. Wieder ist hier in erster Linie die Politik gefragt, da nur ihr die Mittel zur Verfügung stehen, die diesen gewaltigen Aufgaben gewachsen sein könnten.

Aber Politik ist - vor allem unter demokratischen Rahmenbedingungen - oft Ausfluss und Reflex gesellschaftlicher Bedürfnisse, Debatten und Kontroversen. Und insofern eine klimaethische Diskussion, wenn sie den Weg aus den akademischen Kreisen hinaus in die breitere Öffentlichkeit findet, eben auch ein gesellschaftliches Anliegen artikuliert, ist sie durchaus ein Mittel, das Thema Klimaschutz auf die Agenda politischer Akteure zu setzen und ihm dort einen möglichst hohen Stellenwert zu sichern. Wie bereits angedeutet, ist die Politik für dieses Thema bereits sensibilisiert. Und man kann hoffen, dass die Kanzlerin, ihr Kabinett und die nachfolgenden Regierungen bald mit der Eurorettung fertig sein werden und sich dann wieder daran erinnern, dass Deutschland das Zeug dazu hat, eine Vorreiterrolle in der internationalen Klimapolitik zu spielen. Eine klimaethische Diskussion muss jedenfalls nicht in erster Linie etwas zum politischen Thema machen, was bisher kein politisches Thema ist. Ihre gesellschaftlich und politisch wichtigste Funktion liegt wahrscheinlich darin, die haltlosen und offenkundig tendenziösen Entwarnungen einschlägig bekannter Lobbygruppen zu entkräften, die den Leuten erzählen, dass alles nicht gar so schlimm kommen wird. Es liegt auf der Hand, welchen Interessen diese Entwarnungen und Abwiegelungen dienen sollen. Und jeder spürt vermutlich am eigenen Leib ab und an, wie groß die Versuchung ist, dem Sirenengesang der Lobbyisten zu verfallen. Aber trotzdem: Es gibt keinen guten Grund, einfach darauf zu vertrauen, dass vor uns im Nebel keine Kurve liegt. Und unser Fahrzeug wird von Tag zu Tag schneller.

Eine eigenständige Klimaethik könnte sich aber auch deshalb als sinnvoll und nützlich erweisen, weil sich weite Teile des politischen Systems nicht beratungsresistent verhalten, sondern im Gegenteil wissenschaftliche Politikberatung nachfragen. Diese Nachfrage richtet sich derzeit noch vor allem an Umweltökonomen, Klimaforscher und andere Naturwissenschaftler. Aber wie ein Blick in die Sachstandsberichte des Intergovernmental Panel on Climate Change (IPCC) zeigt, ist klimaethische, sprich moralphilosophische Expertise unter den politischen Entscheidungsträgern ein echtes Desiderat. ${ }^{6}$ Zwar wäre es naiv zu glauben, dass globaler Klimaschutz, wenn er denn stattfindet, primär nach moralphilosophischen

6 Ott (2009: 57) weist auf eklatante Mängel in der moralphilosophischen Fundierung des dritten und vierten Sachstandberichts des IPCC hin. 
Gesichtspunkten ausgerichtet sein wird. Macht und Durchsetzungskraft der staatlichen Akteure werden in dieser politischen Großaktion natürlich eine weit gewichtigere Rolle spielen als die Moral oder irgendwelche Theorien der Gerechtigkeit. Aber trotzdem kann man hoffen, dass diese Akteure für die ethischen Implikationen ihres Handelns wenigstens ansatzweise empfänglich sind, wenn es der klimaethischen Diskussion gelingt, diese Implikationen klar und deutlich auszusprechen. Das ist freilich nur eine Hoffnung. Aber ums Hoffen kommen wir ohnehin nicht umhin. Man kann nur hoffen, dass uns am Ende der Straße im Nebel keine Wand erwartet.

\section{Literatur}

Edenhofer, Ottmar/Kowarsch, Martin, 2011: Klimaschutz und Klimawandel, in: Ralf Stoecker/Christian Neuhäuser/Marie-Luise Raters (Hrsg.), Handbuch Angewandte Ethik, Stuttgart/Weimar, 511-517.

Gardiner, Stephen M./Caney, Simon/Jamieson, Dale/Shue, Henry (Hrsg.), 2010:

Climate Ethics. Essential Readings, Oxford.

Gesang, Bernward, 2011: Klimaethik, Berlin.

Krebs, Angelika (Hrsg.), 2000: Gleichheit oder Gerechtigkeit. Texte der neuen Egalitarismuskritik, Frankfurt a. M.

Meyer, Lukas H., 2008: Intergenerational Justice, in: Edward N. Zalta (Hrsg.), Stanford Encyclopedia of Philosophy, http://plato.stanford.edu/entries/justice-intergenerational (Stand: 26.2.2008).

O'Brien, Karen/Clair, Asunción Lera St./Kristoffersen, Berit (Hrsg.), 2010: Climate Change, Ethics, and Human Security, Cambridge.

Ott, Konrad, 2009: Grundzüge der Klimaethik, in: Architektenkammer NordrheinWestfalen (Hrsg.), Natur und gebaute Umwelt, Düsseldorf, 57-64.

Pogge, Thomas/Moellendorf, Darrel (Hrsg.), 2008: Global Justice. Seminal Essays, Saint Paul.

Wallacher, Johannes/Scharpenseel, Karoline (Hrsg.), 2009: Klimawandel und globale Armut, Stuttgart. 
Korrespondenzanschrift:

Prof. Dr. Marco Iorio

Leuphana Universität Lüneburg

C $5 / 020$

Scharnhorststr. 1

21335 Lüneburg

E-Mail: marco.iorio@inkubator.leuphana.de

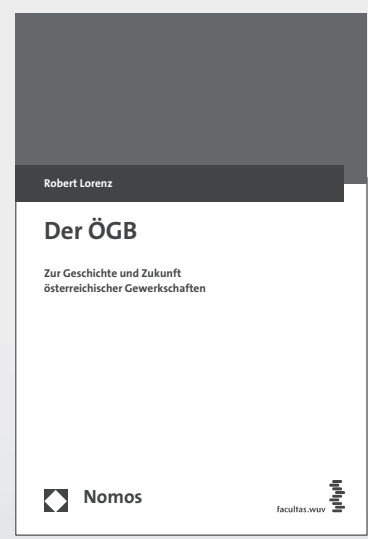

Eine Geschichte über die Krise der österreichischen Gewerkschaften und deren 30 Jahre währenden Versuch der Erneuerung. Einstmals eine der mächtigsten Gewerkschaften der Welt, verlor der ÖGB seit den frühen 1980ern

\section{Der ÖGB}

Zur Geschichte und Zukunft österreichischer Gewerkschaften Von Robert Lorenz

2012, 487 S., brosch., 79,- $€$ ISBN 978-3-8329-7758-0

Mitglieder, Geld und Einfluss. Wie reagierte er darauf und welche Integrationskraft für Demokratie und Gesellschaft ist ihm eigentlich noch zuzutrauen?

Weitere Informationen: www.nomos-shop.de/19561 